\title{
Increased pro-inflammatory milieu in combat related PTSD - a new cohort replication study
}

Daniel Lindqvist ${ }^{1,2}$, Synthia H. Mellon ${ }^{3}$, Firdaus S. Dhabhar ${ }^{4}$, Rachel Yehuda ${ }^{5,6}, \mathrm{~S}$. Marlene Grenon $^{7,8}$, Janine D. Flory ${ }^{5,6}$, Linda M. Bierer ${ }^{5,6}$, Duna Abu-Amara ${ }^{9}$, Michelle Coy $^{1}$, louri Makotkine ${ }^{5,6}$, Victor I. Reus ${ }^{1}$, F. Saverio Bersani ${ }^{10}$, Charles R. Marmar ${ }^{9^{*}}$, Owen M. Wolkowitz ${ }^{1 *}$

${ }^{1}$ Department of Psychiatry, University of California San Francisco (UCSF) School of Medicine, San Francisco, California, United States of America

${ }^{2}$ Lund University, Faculty of Medicine, Department of Clinical Sciences, Lund, Psychiatry, Sweden

${ }^{3}$ Department of OB/GYN and Reproductive Sciences, University of California San Francisco (UCSF)

School of Medicine, San Francisco, California, United States of America

${ }^{4}$ Department of Psychiatry \& Behavioral Sciences, Sylvester Comprehensive Cancer Center, University of Miami, Florida, United States of America

${ }^{5}$ James J. Peters Veterans Administration Medical Center Bronx, New York, United States of America

${ }^{6}$ Icahn School of Medicine at Mount Sinai, New York, New York, United States of America

${ }^{7}$ Department of Surgery, University of California, San Francisco, San Francisco, California, United States of America

${ }^{8}$ Department of Surgery, Veterans Affairs Medical Center, San Francisco, California, United States of America

${ }^{9}$ Steven and Alexandra Cohen Veterans Center for Posttraumatic Stress and Traumatic Brain Injury, Department of Psychiatry, NYU, New York, United States of America

${ }^{10}$ Department of Neurology and Psychiatry, Sapienza University of Rome, Rome, Italy

${ }^{*}$ Correspondence to:

Owen M. Wolkowitz, MD

Department of Psychiatry

University of California, San Francisco (UCSF),

School of Medicine

401 Parnassus Avenue

San Francisco, CA 94143, USA

Phone: +1 415 476-7433

Email: Owen.Wolkowitz@ucsf.edu
Charles R. Marmar, MD

Department of Psychiatry

New York University (NYU) Langone Medical

Center

550 First Avenue New York, NY 10016, USA

Phone: +1 212 263-7300

Email: Charles.Marmar@nyumc.org

KEY WORDS: Post-traumatic stress disorder (PTSD), inflammation, cytokines, depression, combat 


\section{Abstract}

Introduction: Several lines of evidence indicate that increased inflammation is associated with Post-Traumatic Stress Disorder (PTSD). We have previously reported that peripheral inflammatory markers are significantly higher in combatexposed veterans with than without PTSD. This study was designed to replicate these findings in a new study cohort using the same population and recruitment strategies.

Methods: Sixty-one male war veterans (31 PTSD and 30 control subjects) were included in this replication study. Levels of Interleukin-6, Tumor Necrosis Factoralpha, Gamma interferon, and high-sensitivity C-reactive protein were quantified in blood samples. A standardized "total pro-inflammatory score" was calculated to limit the number of statistical comparisons. The Clinician Administered PTSD Scale (CAPS) rating scale was used to assess PTSD symptom severity.

Results: PTSD subjects had significantly higher total pro-inflammatory scores compared to non-PTSD subjects in unadjusted analysis (Cohen's $d=0.75, p=0.005$ ) as well as after adjusting for potentially confounding effects of age, BMI, smoking, and potentially interfering medications and somatic co-morbidities $(p=0.023)$. There were no significant correlations between inflammatory markers and severity of symptoms within the PTSD group.

Conclusions: We replicated, in a new sample, our previous finding of increased inflammatory markers in combat-exposed PTSD subjects compared to combatexposed non-PTSD controls. These findings strongly add to the growing literature suggesting that immune activation may be an important aspect of PTSD pathophysiology, although not directly correlated with current PTSD symptom levels 
in the PTSD group.

$\underline{\text { Introduction }}$

Post Traumatic Stress Disorder (PTSD) is a debilitating condition that places a high burden on patients, their families, and society at large. War veterans are at a particularly high risk of developing PTSD. Between 12 and $18 \%$ of soldiers returning from the conflicts in Iraq and Afghanistan suffer from PTSD (Hoge et al., 2004), and the prevalence rates are even higher in Veterans Affairs healthcare settings (Seal et al., 2009). Biomarkers for PTSD are needed in order to identify subjects at high risk, determine a more accurate diagnosis, track disease progression, better understand the underlying pathophysiology and develop novel treatment options. Several lines of evidence suggest that PTSD is associated with immune activation (Altemus et al., 2003; Passos et al., 2015). Many (Baker et al., 2001; Hoge et al., 2009; Maes et al., 1999; Spitzer et al., 2010), but not all (Bonne et al., 2011), cross-sectional studies have shown that PTSD subjects have significantly higher mean levels of proinflammatory cytokines in blood or cerebrospinal fluid. Further, a recent large-scale genome-wide study reported a significant association between PTSD and $A N K R D 55$, a gene previously linked to autoimmune and inflammatory disorders (Stein et al., 2016).

In line with most of these studies, our group reported that male combat-exposed veterans with PTSD have higher blood levels of pro-inflammatory markers compared to a demographically similar group of combat-exposed male war veterans without 
PTSD (Lindqvist et al., 2014). That study was the first to include a control group comprising combat-exposed individuals who did not develop PTSD, thus taking into account the potential confounding effects of combat exposure per se on cytokine levels. We here report on pro-inflammatory cytokine levels in a replication sample, again comprised of combat-exposed PTSD subjects and combat-exposed controls. Using the same study design, recruitment strategies, and assays as in the prior report (Lindqvist et al., 2014), we hypothesized that a diagnosis of PTSD would again be associated with a pro-inflammatory state in this replication sample.

\section{Material and methods}

\section{Ethical statement}

The Institutional Review Boards of Mt. Sinai School of Medicine (New York, NY), the James J. Peters Veterans Administration Medical Center (Bronx, New York), New York University Medical Center (New York, NY), and the University of California, San Francisco, School of Medicine (San Francisco, CA) approved this study. Study participants gave written and informed consent to participate. The study was conducted in accordance with the provisions of the Helsinki Declaration.

Recruitment procedures and study participants

Subject recruitment procedures and inclusion/exclusion criteria have been described in detail elsewhere (Lindqvist et al., 2014). Briefly, 61 male combat-exposed veterans from Operation Iraqi Freedom and Operation Enduring Freedom were 
recruited by New York University (NYU) and Mt. Sinai/ James J. Peters Veterans Administration (MSSM/JJPVAMC). Diagnoses were established with the Structured Clinical Interview for DSM-IV disorders (First, 1997) and the Clinician Administered PTSD Scale (CAPS) criteria (Blake, 1990). PTSD subjects were positive for current war-zone related PTSD of at least 3 months duration, as indexed by the DSM-IV and the CAPS criteria with CAPS score $>40$. Controls had also served in war zones but were negative for life-time PTSD and had current CAPS score $<20$. Smoking status was determined by self-report or, when this was missing $(n=5,1$ control and 4 PTSD), it was based on plasma cotinine levels.

The CAPS was used to determine the lifetime and current PTSD diagnosis, the severity of current PTSD symptoms (past month), and symptom levels during the most severe lifetime episode of combat-related PTSD. Exposure to early life trauma was evaluated using the Early Trauma Inventory (ETI)- Self Report Short Form (Bremner et al., 2007). Depression symptom severity was assessed with the selfrated Beck Depression Inventory-II (BDI-II) (Beck, 1996). The Deployment Risk and Resilience Inventory-2 (DRRI-2) (Vogt et al., 2013) is a suite of 17 individual scales that assess key deployment-related risk and resilience factors. For the purpose of assessing the degree of combat exposure in PTSD subjects and controls, we used the Combat Experiences subscale (section D). This information was only available in a subset of subjects (25 controls and 21 PTSD subjects). 
Blood sampling and cytokine assays

Blood was drawn in the morning after a night of fasting. Whole blood was collected into $10 \mathrm{ml} \mathrm{SST} \mathrm{tubes} \mathrm{(Becton} \mathrm{Dickinson,} \mathrm{Franklin} \mathrm{Lakes,} \mathrm{NJ).} \mathrm{Serum} \mathrm{levels} \mathrm{of}$ Interleukin (IL)-6, Tumor Necrosis Factor (TNF)- $\alpha$, IL-10 Interferon (IFN)- $\gamma$ and high sensitivity C-reactive protein (hs-CRP) were quantified using previously described assays (Lindqvist et al., 2014). IL-1 $\beta$ was also assayed, however a large number (approximately equally divided between the PTSD and control groups) of values were undetectable, therefore IL-1 $\beta$ was excluded from this report.

In order to minimize the risk of Type I statistical error due to multiple cytokine comparisons (Babyak, 2004), we summarized all the pro-inflammatory cytokine values into a single, "total pro-inflammatory score" by adding standardized z-scores of normalized Interleukin IL-6, TNF- $\alpha$, Interferon (IFN)- $\gamma$, and hs-CRP for each case, similar to the method used in our prior paper (Lindqvist et al., 2014). Exploratory analyses were subsequently conducted on the individual cytokines. One subject had IL-6 below the detection limit of the assay, and in that case we imputed one-half of the lowest detected value. One value for IFN- $\gamma$ was $>10$ SD above the mean and was removed from the subsequent analyses. For the purpose of calculating the total pro-inflammatory score, two missing data points (extreme outlier on IFN- $\gamma$ and CRP) were replaced with mean z-scores of all other available pro-inflammatory cytokine levels for that subject. 
Statistical analyses

Univariate group-wise comparisons were carried out by means of Student's T-test. We controlled for potential confounds by means of analysis of covariance (ANCOVA). Univariate correlations were calculated using Pearson's r. Pearson's chisquare test was used to compare proportions. Before analyses, data were transformed using their natural logarithms (Ln); in cases when log-transformation did not yield a normal distribution, Blom transformation (Blom, 1958) was used.

Covariates in the group comparisons between PTSD and controls were selected based on their known association with cytokines or significant, or near significant, bivariate associations with the total pro-inflammatory score. Based on these criteria the following covariates were used in the ANCOVA's: BMI, smoking and age, and medications and immune/inflammatory illnesses that might affect pro-inflammatory markers. Ten subjects (8 PTSD subjects and 2 controls) either took medications (antidepressants, NSAIDs, statins) or had somatic conditions (asthma, allergies) that might alter cytokine levels.

\section{$\underline{\text { Results }}$}

Demographics and clinical characteristics

Demographic and clinical characteristics of all subjects are presented in Table 1. PTSD subjects were more likely to be smokers $(p=0.031)$. The use of antidepressants was more common in the PTSD group, although not statistically 
significant $(p=0.14)$; this is consistent with the higher proportion of PTSD subjects having comorbid diagnoses of MDD $(p<0.001)$.

Symptom severity scores are shown in Table 1. As expected, subjects with PTSD had significantly higher scores for CAPS current and lifetime symptom severity (all $p<0.001)$, Early Trauma Inventory $(E T I)$ summary score $(p=0.003)$ and BDI-II depression severity scale $(p<0.001)$ compared to controls. PTSD subjects had significantly higher scores on DRRI-2 Combat Experiences subscale $(p<0.001)$, indicating that individuals in this group had been subjected to more severe combat experiences. DRRI-2 combat exposure score did not correlate significantly with total pro-inflammatory score $(\mathrm{p}=0.97)$.

Group comparisons of inflammatory markers levels in PTSD vs. controls

Unadjusted and adjusted group comparisons are summarized in Table 2. PTSD subjects displayed a significantly higher total pro-inflammatory score compared to non-PTSD combat exposed controls in the unadjusted analysis (Cohen's $d=0.75$, $\mathrm{p}=0.005$, Figure 1), as well as after adjusting for age, smoking, BMI and medications/somatic co-morbidities $(p=0.023)$. There was no significant difference in total pro-inflammatory score between PTSD subjects with and without MDD (PTSD+MDD, $0.72 \pm 3.11$; PTSD-MDD, $1.60 \pm 2.81$; Cohen's $d=0.30 ; p=0.44$ ). There was no significant difference in total pro-inflammatory score between smokers and non-smokers $(p=0.23)$. When ETI score was additionally entered as a covariate in the ANCOVA the difference in total pro-inflammatory score between PTSD subjects and non-PTSD subjects was still significant $(p=0.027)$. In the subset in which DRRI-2 
combat exposure score was available, this variable was also entered as a covariate, and this did not change the significant group differences in total pro-inflammatory score $(p=0.033)$.

Exploratory unadjusted analyses of the individual inflammatory markers showed that PTSD subjects also had significantly higher levels of IL-6 $(p=0.003)$ and hs-CRP $(p=0.006)$. Eleven of the PTSD subjects and four of the controls had hs-CRP levels above $3 \mathrm{mg} / \mathrm{L}(\mathrm{p}=0.037)$ indicating an elevated vascular risk in the former group (Ridker, 2003). TNF- $\alpha$ levels were also higher in PTSD subjects at trend level $(p=0.077)$. After adjusting for covariates, the differences in hs-CRP and IL-6 were still statistically significant. There was no significant difference in IFN- $\gamma \square$ or IL$10 \square$ between PTSD subjects and controls.

Correlations between total pro-inflammatory score and clinical symptoms Within the PTSD group, there were no significant correlations between total proinflammatory score and CAPS total current or lifetime scores, BDI, or ETI score (all $\mathrm{p} \geq 0.1)$.

\section{Discussion}

In this study, we replicated in a different set of individuals, our previous finding of increased inflammatory markers in combat-exposed male PTSD subjects compared to combat-exposed non-PTSD male controls (Lindqvist et al., 2014). Specifically, we found that the total pro-inflammatory score, as well as individual inflammatory 
markers IL-6 and hs-CRP, were higher in the PTSD group. This replication further strengthens the notion that immune activation may be an important pathophysiological feature of combat-related PTSD that appears to be independent of combat exposure per se. Further, we also replicated our previous finding that immune activation in PTSD is not significantly correlated with current or lifetime PTSD symptom severity.

Many studies (cited in the introduction) have shown that PTSD subjects, irrespective of the type of trauma, have increased mean levels of pro-inflammatory cytokines. In a recent meta-analysis, IFN- $\gamma \square$ TNF- $\alpha \square \mathrm{IL}-6$, and IL-1 $\beta \square$ wereidentified as the cytokines most commonly elevated in PTSD (Passos et al., 2015). This is mainly in line with the present study, although we found significant between-group differences in a composite "total pro-inflammatory score", an approach that has the advantage of reducing the risk for type 1 statistical error (Babyak, 2004).

Our study adds to the growing body of literature indicating a relationship between PTSD and a pro-inflammatory state. The inclusion of a combat-exposed non-PTSD control group enabled us to take into account the potential confounding effect of combat exposure on cytokine levels. Moreover, we controlled for a number of potential confounders (including age, BMI, smoking, potentially interfering medications and somatic co-morbidities), and our main findings did not substantially change. Only a few previous studies have taken into account the potential mediating effect of co-morbid MDD on the relationship between inflammation and PTSD. This is critical, since MDD, a common co-morbidity of PTSD, may itself be associated with increased inflammation (Dhabhar et al., 2009; Lindqvist et al., 2009; Miller et al., 2009). In the present study, approximately $60 \%$ of the PTSD subjects had co-morbid 
MDD. There was no significant difference in total pro-inflammatory score between PTSD subjects with and without MDD, suggesting that co-morbid MDD was likely not a major driver in the association between PTSD and inflammation. This is in line with our previous report (Lindqvist et al., 2014), as well as studies from other groups (Maes et al., 1999; von Kanel et al., 2010), although not all are in agreement (Gill et al., 2010; Plantinga et al., 2013).

Despite a robust association between PTSD and a pro-inflammatory state, we did not see a correlation between inflammation and PTSD symptom severity within the PTSD group. This is consistent with some previous studies including our own initial report (Lindqvist et al., 2014; Maes et al., 1999), while others have reported such correlations (Gill et al., 2010). Our findings raise the possibility that increased inflammation may be a vulnerability marker for PTSD, as opposed to being the result of trauma exposure or symptom burden. This hypothesis is in line with a large-scale prospective study on male veterans demonstrating that high baseline hs-CRP levels predicted more severe PTSD symptoms post-deployment, even after factoring out trauma exposure (Eraly et al., 2014).

Persons suffering from PTSD are at higher risk of developing a number of somatic illnesses including cardiovascular disease (Coughlin, 2011). Interestingly, increased inflammation has been proposed as a biological mechanism that could confer this increased risk in PTSD subjects (Gander and von Kanel, 2006). Eleven out of 31 PTSD subjects in our sample had hs-CRP levels above $3 \mathrm{mg} / \mathrm{L}$ indicating an elevated vascular risk in this group (Ridker, 2003). Inflammation may increase the risk of cardiovascular disease via interactions with endothelial dysfunction (Grenon 
et al., 2016) and hypercoagulability (von Kanel et al., 2006), two biological characteristics of PTSD.

There are certain limitations to the current study. First, we used an all male sample, thus our findings may not generalize to females with PTSD. Moreover, causality cannot be inferred, given the cross-sectional study design. Thus, longitudinal studies involving cytokine measurements at multiple time points are needed in order to determine whether immune activation in PTSD is a consequence of the disorder or a risk factor. While our use of a combat exposed control group is a strength because it controls for combat exposure per se as a variable affecting inflammatory cytokines, it could also be considered a weakness, since combat exposed individuals who did not develop PTSD may be a resilient group, although we do not have data to further evaluate that possibility. Finally, although our approach of summarizing proinflammatory markers into a standardized total pro-inflammatory score has some distinct advantages as described above, it also comes with some potential shortcomings. E.g., by using a composite score we lose specific information about the individual components (in this case the individual pro-inflammatory markers) while preserving the degrees of freedom in the model (Babyak, 2004). Therefore, we also conducted exploratory analyses on the individual pro-inflammatory markers.

\section{Conclusion}

In this replication study, we affirm that PTSD in male war veterans is associated with a pro-inflammatory state. It is possible that targeting immune-related pathways using pharmacological agents, dietary supplements or life style interventions may be one way forward in improving PTSD clinical management. Furthermore, pro-inflammatory 
cytokines may be used as PTSD biomarkers in order to identify subjects at high risk of inflammation-related illnesses. It is premature, however, to view inflammation as a specific biomarker for PTSD, as inflammation is seen in other psychiatric conditions.

Acknowledgements/Funding:

This study was supported by the following grants: U. S. Department of Defense, W81XWH-11-2-0223 (PI: Charles Marmar); U. S. Department of Defense, W81XWH-10-1-0021 (PI: Owen M. Wolkowitz); The Mental Illness Research, Education and Clinical Center (MIRECC). Daniel Lindqvist was supported by the Swedish Research Council (2015-00387), Marie Sklodowska Curie Actions, Cofund (Project INCA 600398), the Swedish Society of Medicine, the Söderström-Königska Foundation, the Sjöbring Foundation, OM Persson Foundation and the province of Scania (Sweden) state grants (ALF). The authors declare no conflict of interest. The funding sources had no role in in study design; in the collection, analysis and interpretation of data; in the writing of the report; or in the decision to submit the article for publication.

\section{$\underline{\text { References }}$}

Altemus, M., Cloitre, M., Dhabhar, F.S., 2003. Enhanced cellular immune response in women with PTSD related to childhood abuse. Am J Psychiatry 160, 1705-1707.

Babyak, M.A., 2004. What you see may not be what you get: a brief, nontechnical introduction to overfitting in regression-type models. Psychosom Med 66, 411-421.

Baker, D.G., Ekhator, N.N., Kasckow, J.W., Hill, K.K., Zoumakis, E., Dashevsky, B.A., Chrousos, G.P., Geracioti, T.D., Jr., 2001. Plasma and cerebrospinal fluid 
interleukin-6 concentrations in posttraumatic stress disorder.

Neuroimmunomodulation 9, 209-217.

Beck, A.T., Steer, R. A., Brown, G. K, 1996. Manual for the Beck Depression Inventory-II. Psychological Corporation, San Antonio, TX.

Blake, D.D., Weathers, F.W., Nagy, L.M., Kaloupek, D.G., Klauminzer, G., Charney, D.S., Keane, T.M., 1990. A clinician rating scale for assessing current and lifetime PTSD: the CAPS-1. Behav Ther 13, 187-188.

Blom, G., 1958. Statistical estimates and transformed beta-variables. J. Wiley \& sons ; Almqvist \& Wiksell, New York; Stockholm.

Bonne, O., Gill, J.M., Luckenbaugh, D.A., Collins, C., Owens, M.J., Alesci, S., Neumeister, A., Yuan, P., Kinkead, B., Manji, H.K., Charney, D.S., Vythilingam, M., 2011. Corticotropin-releasing factor, interleukin-6, brain-derived neurotrophic factor, insulin-like growth factor-1, and substance $P$ in the cerebrospinal fluid of civilians with posttraumatic stress disorder before and after treatment with paroxetine. J Clin Psychiatry 72, 1124-1128.

Bremner, J.D., Bolus, R., Mayer, E.A., 2007. Psychometric properties of the Early Trauma Inventory-Self Report. J Nerv Ment Dis 195, 211-218.

Coughlin, S.S., 2011. Post-traumatic Stress Disorder and Cardiovascular Disease. Open Cardiovasc Med J 5, 164-170.

Dhabhar, F.S., Burke, H.M., Epel, E.S., Mellon, S.H., Rosser, R., Reus, V.I., Wolkowitz, O.M., 2009. Low serum IL-10 concentrations and loss of regulatory 
association between IL-6 and IL-10 in adults with major depression. J Psychiatr Res 43, 962-969.

Eraly, S.A., Nievergelt, C.M., Maihofer, A.X., Barkauskas, D.A., Biswas, N., Agorastos, A., O'Connor, D.T., Baker, D.G., Marine Resiliency Study, T., 2014. Assessment of plasma C-reactive protein as a biomarker of posttraumatic stress disorder risk. JAMA Psychiatry 71, 423-431.

First, M.B., 1997. Structured clinical interview for DSM-IV axis I disorders SCID-I : clinician version, administration booklet. American Psychiatric Press, Washington, DC.

Gander, M.L., von Kanel, R., 2006. Myocardial infarction and post-traumatic stress disorder: frequency, outcome, and atherosclerotic mechanisms. Eur J Cardiovasc Prev Rehabil 13, 165-172.

Gill, J., Luckenbaugh, D., Charney, D., Vythilingam, M., 2010. Sustained elevation of serum interleukin- 6 and relative insensitivity to hydrocortisone differentiates posttraumatic stress disorder with and without depression. Biological psychiatry 68, 999-1006.

Grenon, S.M., Owens, C.D., Alley, H., Perez, S., Whooley, M.A., Neylan, T.C., Aschbacher, K., Gasper, W.J., Hilton, J.F., Cohen, B.E., 2016. Posttraumatic Stress Disorder Is Associated With Worse Endothelial Function Among Veterans. J Am Heart Assoc 5.

Hoge, C.W., Castro, C.A., Messer, S.C., McGurk, D., Cotting, D.I., Koffman, R.L., 2004. Combat duty in Iraq and Afghanistan, mental health problems, and barriers to care. N Engl J Med 351, 13-22. 
Hoge, E.A., Brandstetter, K., Moshier, S., Pollack, M.H., Wong, K.K., Simon, N.M., 2009. Broad spectrum of cytokine abnormalities in panic disorder and posttraumatic stress disorder. Depress Anxiety 26, 447-455.

Lindqvist, D., Janelidze, S., Hagell, P., Erhardt, S., Samuelsson, M., Minthon, L., Hansson, O., Bjorkqvist, M., Traskman-Bendz, L., Brundin, L., 2009. Interleukin-6 is elevated in the cerebrospinal fluid of suicide attempters and related to symptom severity. Biological psychiatry 66, 287-292.

Lindqvist, D., Wolkowitz, O.M., Mellon, S., Yehuda, R., Flory, J.D., Henn-Haase, C., Bierer, L.M., Abu-Amara, D., Coy, M., Neylan, T.C., Makotkine, I., Reus, V.I., Yan, X., Taylor, N.M., Marmar, C.R., Dhabhar, F.S., 2014. Proinflammatory milieu in combat-related PTSD is independent of depression and early life stress. Brain, behavior, and immunity 42, 81-88.

Maes, M., Lin, A.H., Delmeire, L., Van Gastel, A., Kenis, G., De Jongh, R., Bosmans, E., 1999. Elevated serum interleukin-6 (IL-6) and IL-6 receptor concentrations in posttraumatic stress disorder following accidental man-made traumatic events. Biological psychiatry 45, 833-839.

Miller, A.H., Maletic, V., Raison, C.L., 2009. Inflammation and its discontents: the role of cytokines in the pathophysiology of major depression. Biological psychiatry $65,732-741$.

Passos, I.C., Vasconcelos-Moreno, M.P., Costa, L.G., Kunz, M., Brietzke, E., Quevedo, J., Salum, G., Magalhaes, P.V., Kapczinski, F., Kauer-Sant'Anna, M., 2015. Inflammatory markers in post-traumatic stress disorder: a systematic review, meta-analysis, and meta-regression. Lancet Psychiatry 2, 1002-1012. 
Plantinga, L., Bremner, J.D., Miller, A.H., Jones, D.P., Veledar, E., Goldberg, J., Vaccarino, V., 2013. Association between posttraumatic stress disorder and inflammation: a twin study. Brain, behavior, and immunity 30, 125-132.

Ridker, P.M., 2003. Cardiology Patient Page. C-reactive protein: a simple test to help predict risk of heart attack and stroke. Circulation 108, e81-85.

Seal, K.H., Metzler, T.J., Gima, K.S., Bertenthal, D., Maguen, S., Marmar, C.R., 2009. Trends and risk factors for mental health diagnoses among Iraq and Afghanistan veterans using Department of Veterans Affairs health care, 2002-2008. American journal of public health 99, 1651-1658.

Spitzer, C., Barnow, S., Volzke, H., Wallaschofski, H., John, U., Freyberger, H.J., Lowe, B., Grabe, H.J., 2010. Association of posttraumatic stress disorder with lowgrade elevation of C-reactive protein: evidence from the general population. $\mathrm{J}$ Psychiatr Res 44, 15-21.

Stein, M.B., Chen, C.Y., Ursano, R.J., Cai, T., Gelernter, J., Heeringa, S.G., Jain, S., Jensen, K.P., Maihofer, A.X., Mitchell, C., Nievergelt, C.M., Nock, M.K., Neale, B.M., Polimanti, R., Ripke, S., Sun, X., Thomas, M.L., Wang, Q., Ware, E.B., Borja, S., Kessler, R.C., Smoller, J.W., Army Study to Assess, R., Resilience in Servicemembers, C., 2016. Genome-wide Association Studies of Posttraumatic Stress Disorder in 2 Cohorts of US Army Soldiers. JAMA Psychiatry.

Vogt, D., Smith, B.N., King, L.A., King, D.W., Knight, J., Vasterling, J.J., 2013. Deployment risk and resilience inventory-2 (DRRI-2): an updated tool for assessing psychosocial risk and resilience factors among service members and veterans. $\mathrm{J}$ Trauma Stress 26, 710-717. 
von Kanel, R., Begre, S., Abbas, C.C., Saner, H., Gander, M.L., Schmid, J.P., 2010. Inflammatory biomarkers in patients with posttraumatic stress disorder caused by myocardial infarction and the role of depressive symptoms. Neuroimmunomodulation $17,39-46$.

von Kanel, R., Hepp, U., Buddeberg, C., Keel, M., Mica, L., Aschbacher, K., Schnyder, U., 2006. Altered blood coagulation in patients with posttraumatic stress disorder. Psychosom Med 68, 598-604.

\section{Figure legend}

Figure 1. Total pro-inflammatory score in PTSD subjects and controls. Error bars indicate +/- 1 SD from the mean. The total pro-inflammatory score is given as a standardized score; thus, positive or negative values may be seen. 


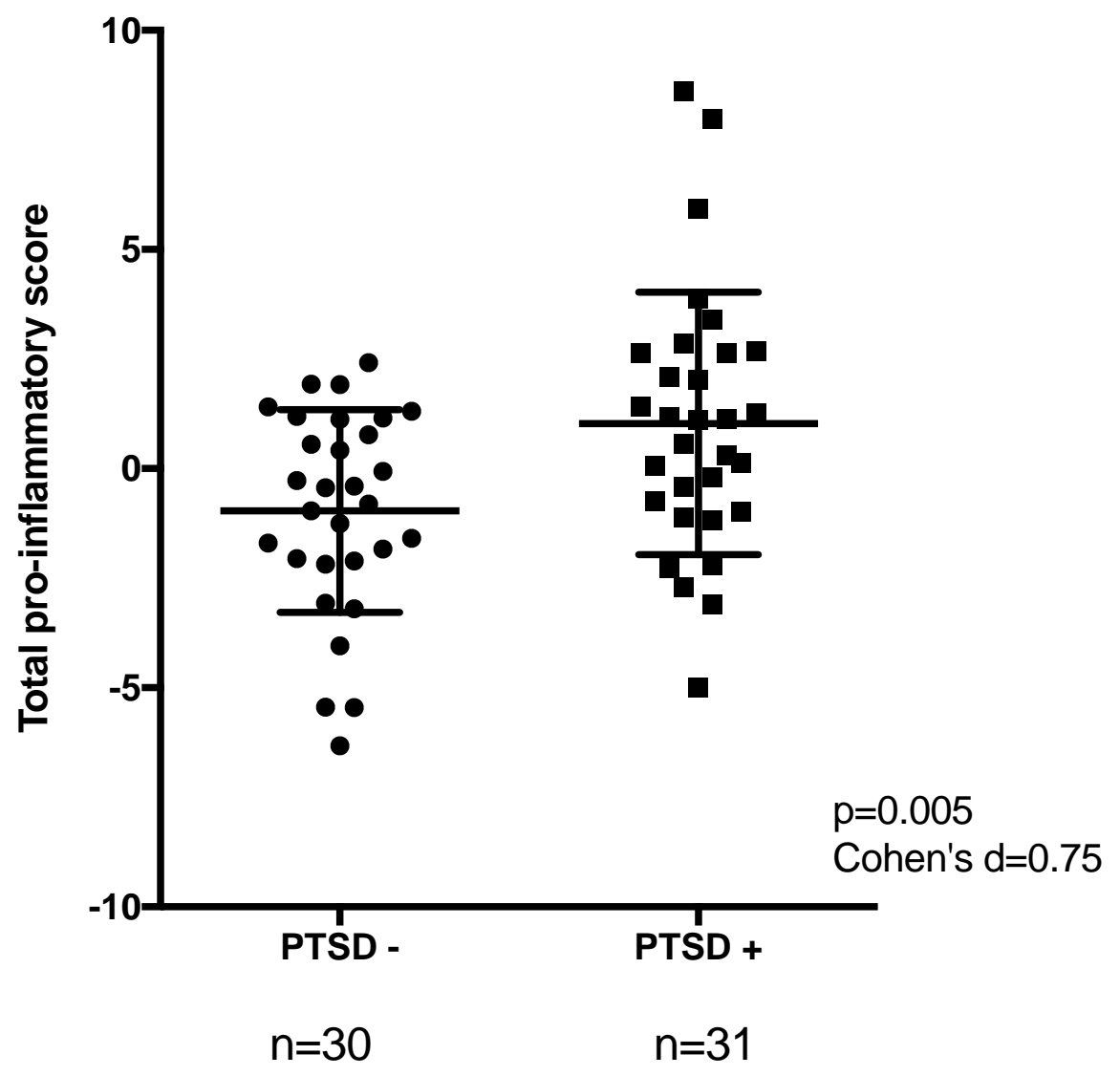

